\title{
Réflexions sur la température moyenne de la planète
}

\author{
Olivier Boucher', Marie Doutriaux-Boucher ${ }^{2}$, Jean-Louis Dufresne ${ }^{3}$ \\ 1. Institut Pierre-Simon Laplace, CNRS / Sorbonne Université, Paris \\ 2. Eumetsat, Darmstadt, Allemagne \\ 3. Laboratoire de météorologie dynamique, CNRS / Sorbonne Université, Paris \\ olivier.boucher@ipsl.fr
}

But pédagogique : engager une réflexion avec le public ou les étudiants sur la notion de température moyenne de la planète, les difficultés à la définir et la mesurer, mais aussi la robustesse du réchauffement planétaire observé depuis 1850 .

Public: tout public, discours adaptable.

Matériel nécessaire: plusieurs thermomètres à infrarouge ou à thermocouple.

Sites internet: les étudiants peuvent être dirigés vers les sites suivants pour approfondir le sujet: https:// crudata.uea.ac.uk/cru/data/temperature/, https://www.ncdc.noaa.gov/sotc/global/, https://data.giss.nasa.gov/gistemp/, http://berkeleyearth.org/ et http:// www.surfacetemperatures.org/.
$1 \mathrm{y}$ a fort à parier que toute personne intéressée par le climat que l'on peut croiser lors de séminaires destinés au grand public ou de « fêtes de la science » aura déjà vu une courbe d'évolution de la température moyenne de la planète du type de celle présentée sur la figure 1. Celle que 1'on a sélectionnée ici provient du $5^{\mathrm{e}}$ rapport du Groupe intergouvernemental d'experts sur l'évolution du climat (Giec). Elle montre l'anomalie de la température annuelle globale par rapport à la climatologie, prise ici comme la moyenne des 30 années allant de 1961 à 1990. Cette courbe combine les températures de surface de 1'air (aussi appelée t $2 \mathrm{~m}$ pour température à 2 mètres) au-dessus des continents et les températures de surface de l'océan (c'est-à-dire la température de l'eau, sea surface temperature ou SST en anglais). On y voit trois courbes correspondant à des traitements statistiques différents - par

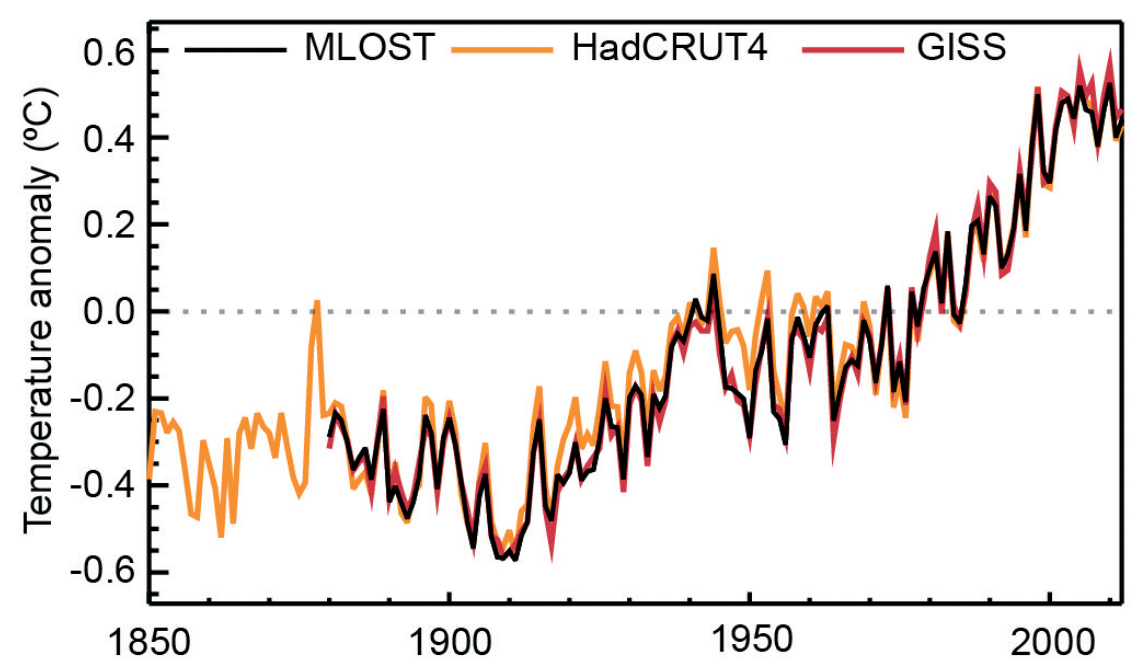

Figure 1. Anomalies de la température moyenne annuelle globale ( ${ }^{\circ} \mathrm{C}$ ) par rapport à la période 1961-1990 selon trois traitements statistiques de jeux de données (HadCRUT4, GISS et NCDC MLOST) combinant les températures de l'air de surface sur les continents et les températures de surface de l'océan. Source : Hartmann et al. (2013). différentes équipes de recherche d'essentiellement les mêmes jeux de données. Les températures sont des anomalies par rapport à la moyenne climatologique des années 1961 à 1990, c'est-à-dire que les courbes ne renseignent pas sur la température moyenne de la planète, mais sur l'évolution de celle-ci entre 1850 et 2011. On y voit que les trois traitements statistiques donnent des résultats très similaires, qu'il y a un réchauffement de l'ordre de $0,8{ }^{\circ} \mathrm{C}$ entre 1900 et 2011 et que ce réchauffement n'est pas continu dans le temps ${ }^{1}$. On peut aussi noter qu'aucune barre d'incertitude n'est présentée sur la figure 1, on reviendra sur ce point plus tard.

Il est légitime de se poser la question du rôle et de la fiabilité des traitements statistiques qui se cachent derrière une telle courbe. En effet, différents groupes de recherche peuvent faire des choix différents et il faut reconnaître une part de subjectivité dans ceuxci. Le but de cet article (et de l'échange avec les étudiants ou le public) est de procéder par analogie avec la température moyenne d'une pièce et d'engager une réflexion sur la notion de température moyenne de la planète, les difficultés à la définir et à la mesurer, mais aussi la robustesse du réchauffement planétaire observé depuis le début du siècle dernier.

1. Il y a plusieurs manières de quantifier l'augmentation de température sur une période donnée compte tenu de la variabilité naturelle. Il $n$ 'est pas pertinent de comparer une année récente précise (par exemple 2011) avec une année ancienne précise (1900). On peut comparer la moyenne sur une période récente (par exemple 1982-2011) avec une période ancienne (par exemple 1900-1929) ou alors estimer l'augmentation de température à partir de la pente d'une régression linéaire sur toute la période. 


\section{Analogie avec la température de la pièce}

Pour initier la discussion, on propose de mesurer la température dans une pièce avec au moins deux thermomètres qui pourront être des thermomètres à infrarouge ou des thermomètres à sonde de température. Les thermomètres à infrarouge (figure 2a) étant souvent équipés d'un laser pour illuminer le point dont on mesure la température, il convient de rappeler qu'il ne faut pas viser son œil ou celui du voisin. Le thermomètre à infrarouge, dont on peut rappeler le principe (Dubuisson et Minvielle, 2017), ne mesure pas la température de l'air, mais celle d'une surface (un petit morceau du mur d'une pièce par exemple). L'exercice consistera à essayer de définir et de mesurer la température moyenne des murs (et du plafond et des sols) de la pièce dans laquelle on se situe.

Pour l'autre type de thermomètre, la sonde est souvent l'extrémité d'un thermocouple qui peut être apparente ou protégée (figure 3 ). Cette extrémité est en contact avec l'air avec lequel elle échange de l'énergie, mais elle reçoit également du rayonnement infrarouge émis par les murs et éventuellement du rayonnement solaire. La température de l'extrémité du thermocouple n'est donc pas exactement égale à celle de l'air. C'est ce type de capteur qui est utilisé dans les stations météorologique et c'est pour cela qu'ils sont placés dans des abris qui les protègent du rayonnement solaire. On privilégiera des thermomètres qui ont un temps de réponse court pour que l'expérience soit plus dynamique. L'exercice consistera à mesurer la température en une dizaine de points de la pièce, de manière à pouvoir déterminer la température moyenne mais aussi la dispersion des mesures ponctuelles dont l'écart à la moyenne peut atteindre quelques degrés Celsius.

\section{Fidélité, exactitude et correction des biais}

Dans un premier temps, on s'intéresse à la température en un point donné et on reviendra plus tard sur les problèmes d'échantillonnage spatial. Il est utile de
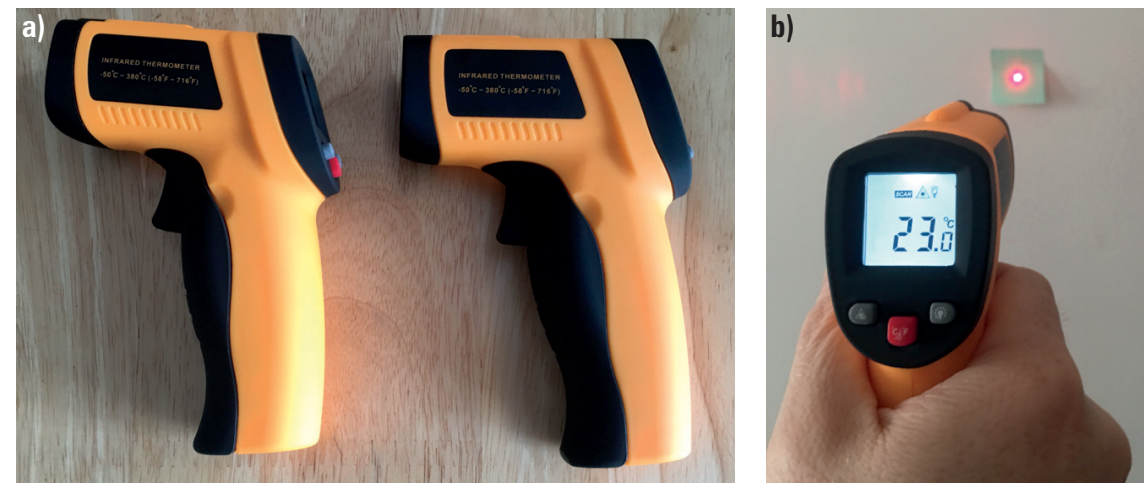

Figure 2. a) Photographie des deux thermomètres à infrarouge nécessaires à l'expérience, b) exemple de mesure de la température sur un point du mur identifié par un morceau de papier.

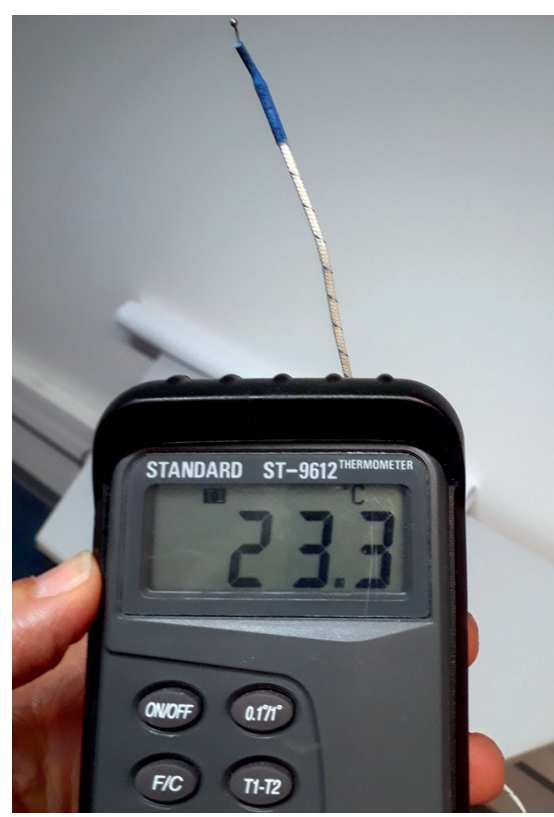

Figure 3. Exemple de mesure de la température de l'air avec un thermomètre à thermocouple.

définir les notions de fidélité et d'exactitude de la mesure (voir encadré « Glossaire »). La figure 4 illustre ces notions : la fidélité se réfère à la répétabilité (ou reproductibilité) de la mesure. Autrement dit, trouve-t-on le même résultat si on répète plusieurs fois la mesure (à supposer bien entendu que la mesure ne perturbe pas la variable que l'on cherche à mesurer) ? L'exactitude, quant à elle, représente l'écart entre la mesure et la "vraie » valeur de la variable que l'on cherche à mesurer. Autrement dit, la mesure est exacte s'il n'y a pas de biais ou d'erreur systématique. Nos deux thermomètres à infrarouge nous permettent d'appréhender ces deux notions de manière concrète.

On peut facilement quantifier la fidélité de la mesure en répétant un grand nombre de fois la même mesure avec un thermomètre donné (figure $2 b$ et tableau 1). On constate qu'un même thermomètre ne donne pas toujours la même valeur de température pour la même cible, quand la mesure est répétée plusieurs fois. On admet assez facilement que la vraie température du mur ne varie pas aussi rapidement et qu'il s'agit d'un artefact dû à nos instruments de mesure. Dans l'exemple du tableau 1, l'écart type de la mesure est de $0,23{ }^{\circ} \mathrm{C}$ pour le thermomètre $\mathrm{A}$ et de $0,37^{\circ} \mathrm{C}$ pour le thermomètre $\mathrm{B}$. Lécart type nous donne une idée de la fidélité de la mesure réalisée. Cela dit, la fidélité n'est pas vraiment un problème dans notre cas si on souhaite

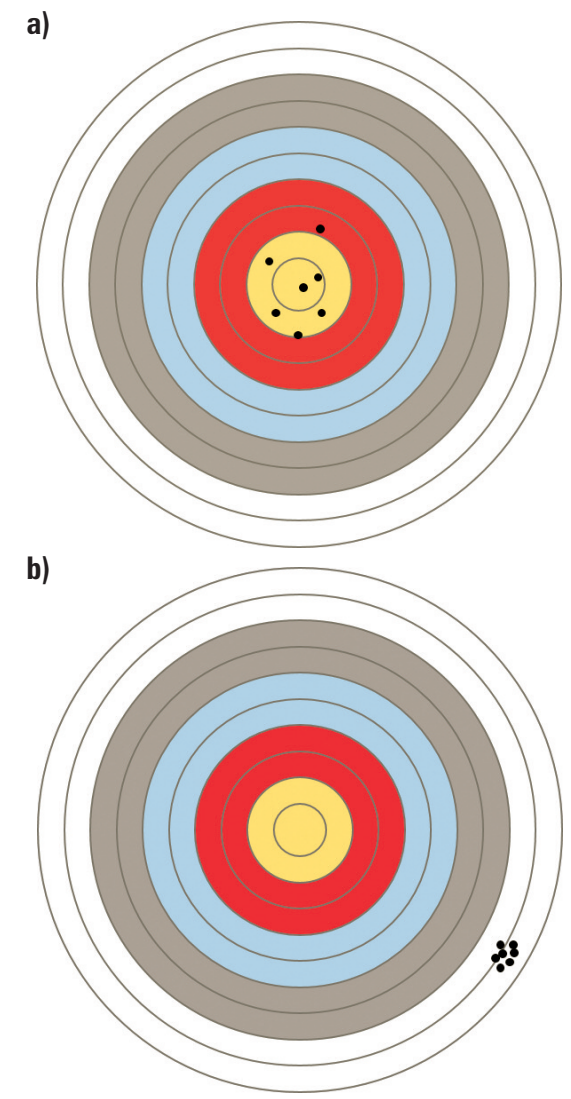

Figure 4. Schéma illustrant la différence entre fidélité et exactitude. a) Les sept tirs se rapprochent tous du centre de la cible ; ils témoignent de peu de fidélité, mais d'une bonne exactitude. b) Les sept tirs se situent tous très proches d'un même point éloigné du centre de la cible ; ils témoignent de peu d'exactitude mais d'une bonne fidélité. 


\section{Glossaire}

Anomalie (anomaly) : différence entre la valeur d'une grandeur physique et la valeur moyenne climatologique de cette même grandeur physique estimée sur une période de référence (en général de 30 ans). On travaille souvent en anomalie de température pour s'affranchir de l'incertitude sur la valeur climatologique et pour minimiser les problèmes induits par l'ajout ou le retrait de stations dans le réseau de mesure.

Biais (bias) ou erreur systématique (systematic error) : différence entre la valeur moyenne mesurée et la vraie valeur moyenne d'une grandeur physique. Le biais est lié à l'exactitude de la mesure. On peut distinguer plusieurs sources de biais, en particulier le biais de mesure dû à une technique de mesure imparfaite et le biais d'échantillonnage dû à un échantillonnage spatial ou temporel inapproprié. L'estimation de la température moyenne du globe est sujette à ces deux types de biais, de manière variable dans le temps, en raison de changements dans l'instrumentation et les réseaux de mesure. On peut distinguer le biais de couverture dû à une région non prise en compte au biais d'échantillonnage lui-même.

Correction de biais (bias correction) : méthode qui consiste à corriger les biais (ou erreurs systématiques) qui ont été identifiés et quantifiés. II existe un grand nombre de méthodes selon la grandeur physique et le type de biais que l'on cherche à corriger. obtenir la température moyenne du morceau de papier et que l'on dispose d'un grand nombre de mesures. Les erreurs se compensent entre elles. Le plus important est de savoir si la mesure est exacte. Il est difficile, voire impossible, de répondre à cette question car nous n'avons pas accès à la « vraie » température du morceau de papier. Par contre, le fait que les deux thermomètres donnent des résultats différents (moyennes respectives de 22,6 et $21,9{ }^{\circ} \mathrm{C}$ ) nous permet de dire que la mesure d'au moins l'un des deux n'est pas exacte à $0,1^{\circ} \mathrm{C}$ près, et il est plus que probable qu'aucune des deux ne l'est !

Si, par analogie avec la température de la planète, on s'intéresse aux variations de température dans le temps (c'est-à-

Tableau 1. Statistiques (nombre de mesures, médiane, moyenne et écart type) de la mesure de la température du papier avec deux thermomètres à infrarouge.

\begin{tabular}{lcc} 
& $\begin{array}{c}\text { Thermomètre } \\
\mathbf{A}\end{array}$ & $\begin{array}{c}\text { Thermomètre } \\
\text { B }\end{array}$ \\
\hline Nombre de mesures & 20 & 20 \\
\hline Médiane & $22,6^{\circ} \mathrm{C}$ & $22.0^{\circ} \mathrm{C}$ \\
\hline Moyenne & $22,6^{\circ} \mathrm{C}$ & $21,9^{\circ} \mathrm{C}$ \\
\hline Écart type & $0,23^{\circ} \mathrm{C}$ & $0,37^{\circ} \mathrm{C}$ \\
\hline
\end{tabular}

Échantillonnage (sampling) : procédure qui consiste à sélectionner un échantillon, c'est-àdire un nombre fini de points de mesure et/ou de mesures dans le contexte de cet article. En général, cette procédure est définie de sorte que les points de mesure sélectionnés soient les plus représentatifs possibles des propriétés de l'ensemble pour une variable donnée. Dans cet article, on parle de sous-échantillonnage lorsque l'on considère que la procédure ne satisfait pas complètement ce critère de représentativité. C'est notamment le cas dans les régions relativement pauvres en mesures (régions polaires, Afrique, océans de I'hémisphère Sud).

Exactitude (accuracy) : étroitesse de l'accord entre la valeur mesurée et la valeur vraie de la grandeur physique que l'on cherche à mesurer. Définition adaptée de BIPM (2012).

Fidélité (precision) : étroitesse de l'accord entre les valeurs mesurées obtenues par des mesures répétées de la même grandeur physique. Définition adaptée de BIPM (2012).

Incertitude (uncertainty) : dispersion des valeurs attribuées à une grandeur physique que l'on cherche à mesurer. Définition adaptée de BIPM (2012).

Précision : le mot précision étant ambigu en français, nous suivons les recommandations $d u$ BIPM (2012) et n'utilisons pas ce terme dans cet article.

Voir BIPM (2012) pour un exposé complet du vocabulaire utilisé en métrologie. beaucoup de discussion sur les causes potentielles des biais : changements d'instruments, changements d'abris sur les continents, changements dans l'environnement des stations (arbres, bâtiments...), changements dans la méthode de prélèvement de l'eau pour les mesures de SST...

\section{Échantillonnage}

Après avoir évoqué les corrections de biais, nous pouvons revenir à la question de comment calculer la température moyenne de la planète en procédant par analogie avec la température moyenne des murs de la pièce (ou de l'air de la pièce dans le cas où on utilise un thermomètre à thermocouple). Comme on ne peut mesurer la température de chaque point des murs, on comprend bien intuitivement qu'il faille échantillonner différents points des murs. Si on travaille avec les thermomètres à infrarouge, on peut par exemple diviser la surface du mur en petits rectangles réguliers et effectuer une mesure ponctuelle dans chaque rectangle. La densité du maillage dépendra des variations attendues de la température les murs. Si la température est homogène, seuls quelques points de mesure suffiront. Si la température est peu homogène (présence d'un radiateur ou d'une fenêtre), il sera judicieux de resserrer le maillage. Au final, le maillage choisi dépendra de la connaissance a priori que l'on a du milieu que l'on observe, mais aussi de considérations pratiques. La température moyenne des murs de la pièce correspondra à la moyenne des températures des différents murs pondérées par leurs surfaces respectives, mais il y a peut-être des parties du mur que l'on ne peut atteindre facilement (derrière un meuble ou un radiateur).

Sur les océans, on mesure le plus souvent non pas la température de l'air, mais la température de l'eau de surface. Les conditions de prélèvement ont elles aussi changé au cours du temps. L'exactitude des mesures (ou autrement dit leur biais) a donc évolué dans le temps ; la principale tâche des groupes travaillant sur les données de température consiste à corriger les biais relatifs des différents principes et instruments de mesure utilisés depuis 1850 (Moisselin et al., 2002 ; Kent et al., 2017). Les méthodes d'homogénéisation font l'objet de travaux de recherche et continuent d'évoluer ${ }^{4}$. La question soulevée en TD auprès d'étudiants en $3^{\mathrm{e}}$ année universitaire génère souvent
Nous avons là un excellent analogue avec la température moyenne de la planète. En effet, la distribution des stations météorologiques sur les continents (figure 5) et celle des

2. Voir http://education.meteofrance.fr/ressourcespour-les-enseignants/observer-et-mesurer/latemperature1

3. Voir http://education.meteofrance.fr/ressourcespour-les-enseignants/observer-et-mesurer/l-abrimeteo

4. On peut signaler l'International Surface Temperature Initiative qui vise à comparer les méthodes d'homogénéisation entre elles à partir d'un jeu de données synthétiques auquel on a incorporé des ruptures d'homogénéité. Voir http://www.surfacetemperatures.org/ 
bateaux sur les océans (non montré) sont loin d'être homogènes. Certaines régions continentales (Amérique du Nord, Europe, une partie de l'Asie) sont très bien échantillonnées, alors que d'autres (régions polaires, Afrique, Amazonie) sont sous-échantillonnées. De plus, les mesures étaient peu denses en 1850 et le nombre de stations a augmenté au cours du temps. C'est donc là la deuxième difficulté à laquelle se heurtent les différentes équipes de recherche qui travaillent sur la question.

Cette difficulté n'est cependant pas insurmontable. À la surface de l'océan, la température est très homogène spatialement, un nombre assez faible de mesures permet d'estimer avec une bonne exactitude la température moyenne. À la surface des continents, la température peut varier de plusieurs degrés sur des courtes distances, mais la latitude, l'altitude et la distance à l'océan expliquent une très grande partie des variations observées sur la valeur climatologique de la température et la densité d'observation est en général suffisamment élevée, à l'exception des régions mentionnées cidessus. Enfin, les régions couvertes de glace de mer posent un problème particulier sur lequel nous reviendrons.

Une solution au problème de souséchantillonnage consiste à laisser des « trous » dans le calcul de la moyenne planétaire, une autre à extrapoler les données existantes au-dessus des régions mal échantillonnées et une troisième à remplir les « trous » par des mesures satellitaires de la température de surface. Aucune des solutions n'est complètement satisfaisante et il est important que plusieurs solutions cohabitent. Cependant, l'impact sur le résultat reste faible. Cowtan et Way (2014) ont estimé des biais de couverture sur la température moyenne du globe de l'ordre de quelques centièmes de degré Celsius pour HadCRUT4 par rapport aux autres jeux de données.

Ce problème de sous-échantillonnage ne serait pas très grave si toutes les régions du monde se réchauffaient à la même vitesse, mais il se trouve que les régions polaires sous-échantillonnées sont aussi celles qui se réchauffent le plus vite. Il est donc important d'essayer de corriger cet effet le mieux possible. L'impact d'un sous-échantillonnage dépend aussi de l'échelle de temps considérée. Plus on s'intéresse à une échelle de temps longue, moins le sous-échantillonnage est un problème, et inversement.

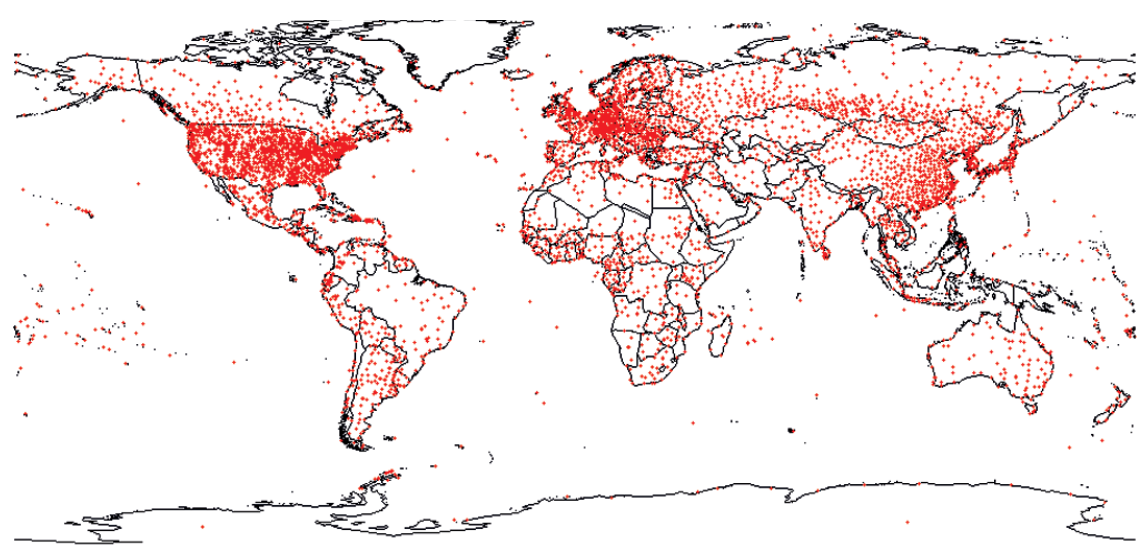

Figure 5. Emplacements des quelque 6000 stations de mesure de la température ayant contribué à la version 1 du jeu de données Global Historical Climatology Network (GHCN). Source https://daac.ornl.gov/CLIMATE/guides/CDIAC NDP41.html.
Des méthodes statistiques sophistiquées permettent de quantifier l'incertitude autour de la moyenne de la planète. Celle-ci augmente au fur et à mesure qu'on remonte dans le temps et que l'échantillonnage se détériore, mais reste faible jusqu'en 1850 (figure 6, Rohde et al., 2013a, b). C'est pourquoi les différentes courbes présentées sur la figure 1 sont très proches les unes des autres. Elles ont été récemment rejointes par une étude indépendante réalisée par le projet Berkeley Earth ${ }^{5}$. Il est intéressant de retracer l'historique de ce projet, né à l'initiative de physiciens de la prestigieuse université californienne de Berkeley. Il fait suite à l'épisode du ClimateGate qui trouve son origine dans le piratage de la messagerie du Climatic Research Unit en Grande-Bretagne. Des extraits de messages ont été publiés, qui ont pu laisser croire, sortis de leur contexte, que certaines données de température avaient été manipulées. Des physiciens de Berkeley ont ouvertement remis en cause la façon de travailler des climatologues et leurs résultats, et ont lancé un projet visant à faire, cette foisci « sérieusement» (sous-entendu par des gens sérieux), une nouvelle analyse des températures mesurées à la surface des continents. Leurs premiers résultats, publiés à partir de l'année 2013, ont confirmé que les mesures réalisées à la surface de la Terre indiquaient bien un réchauffement global de la planète, avec des valeurs

5. http://berkeleyearth.org/land-and-ocean-data/

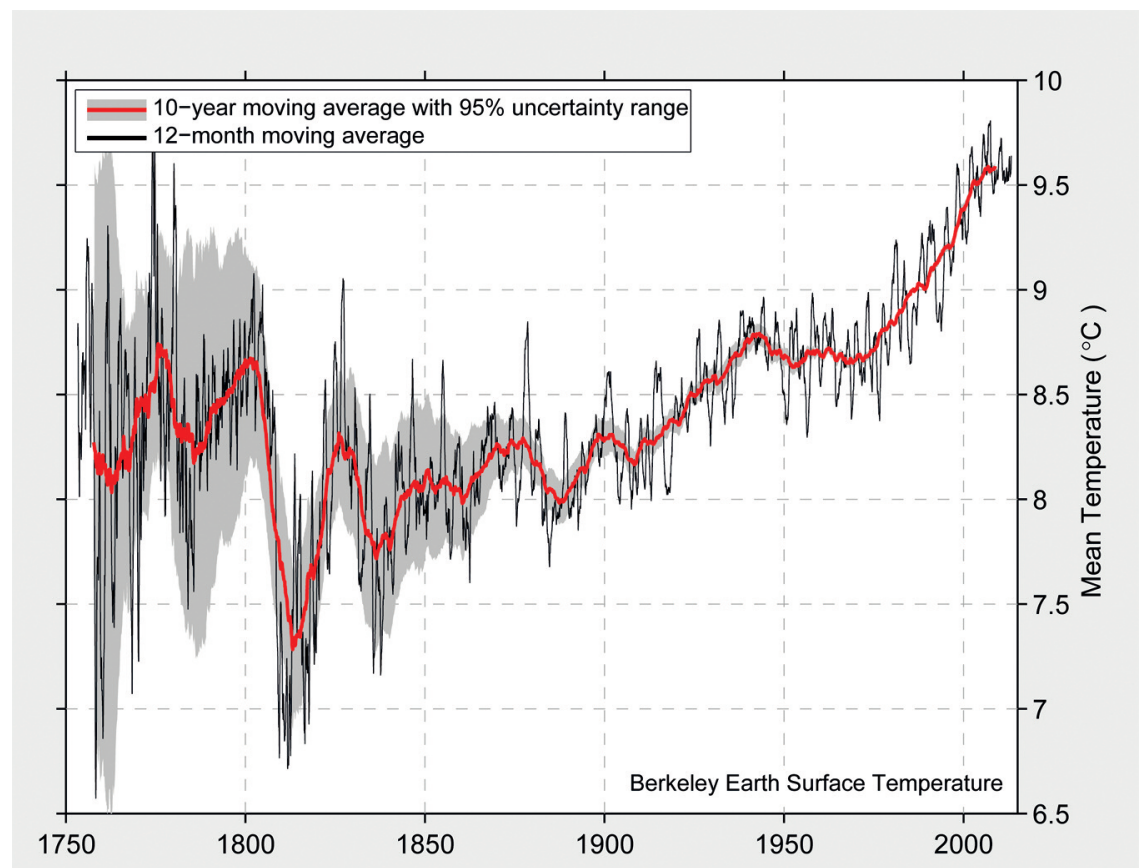

Figure 6. Variations de la température moyenne sur les continents avec une moyenne glissante de 12 mois (en noir) ou de 10 ans (en rouge). La courbe rouge est accompagnée d'un intervalle de confiance (plage en gris) représentant une incertitude de $95 \%$. Source http://berkeleyearth.lbl.gov/regions/global-land 
très proches des résultats des études précédentes. Les données et les programmes utilisés ont été rendus publics, rendant la démarche très transparente, comme les auteurs s'y étaient engagés. Ce projet, initialement polémique, a eu le grand mérite d'être mené jusqu'au bout, de montrer que les résultats obtenus par les climatologues étaient effectivement robustes et ainsi de faire taire un certain nombre de critiques injustifiées et stériles.

On peut néanmoins signaler une difficulté quand il s'agit de « combiner » les températures de surface au-dessus des continents et celles des océans. En effet, on cherche là à combiner deux quantités différentes : température de l'air et température de l'eau de surface. Cette opération reste légitime, car on peut considérer que la température de surface de l'air au-dessus des océans est en moyenne à l'équilibre avec la température de l'eau de surface, ce que nous confirment les modèles météorologiques et les modèles de climat. De plus, on travaille en anomalie de température par rapport à une climatologie. Une question se pose sur comment traiter les régions de glace de mer, dont la couverture varie au cours du temps : température de l'air au-dessus de la glace ou température de la mer sous la glace. Berkeley Earth a essayé les deux solutions avec au final très peu de différence sur l'évolution de la température moyenne de la planète. La première méthode donne une augmentation de température entre 1850 et 2014 de $0,06{ }^{\circ} \mathrm{C}$ plus élevée que celle de la deuxième méthode du fait de la fonte de la glace de mer.
Une dernière remarque concerne un argument parfois avancé par les "climato-sceptiques" sur la température moyenne de la planète qui ne serait pas une variable physiquement pertinente. Mathématiquement ou statistiquement parlant, rien n'empêche bien sûr de faire la moyenne spatiale ou temporelle de la température de surface. Physiquement parlant, il est vrai que la température est une variable thermodynamique intensive. Même multipliée par une surface ou un volume élémentaire, elle n'est pas additive et il peut apparaître incongru d'en faire la moyenne. Là aussi, il existe une explication très simple. Le produit de la capacité thermique (de l'air ou de l'eau) et de la température est homogène à une énergie. Or l'énergie est une variable extensive que l'on peut additionner et moyenner. Les variations de la capacité thermique (de l'air ou de l'eau) sont très faibles : celle de l'air est comprise entre 1003 et $1006 \mathrm{~J} \mathrm{~kg}^{-1} \mathrm{~K}^{-1}$ pour la gamme de températures rencontrées dans l'atmosphère, alors que celle de l'eau liquide est comprise entre 4217 et $4178 \mathrm{~J} \mathrm{~kg}^{-1} \mathrm{~K}^{-1}$ quand la température varie entre 0 et $30^{\circ} \mathrm{C}$. Il est donc légitime de calculer une température moyenne planétaire qui correspond finalement à la température équivalente de l'énergie moyenne de la couche de surface de l'atmosphère ou de l'océan. On peut aussi noter que la température moyenne de la planète calculée ainsi est proche mais n'est pas tout à fait physiquement équivalente aux températures radiatives équivalentes estimées avec des modèles simplifiés du bilan radiatif (avec ou sans effet de serre) du fait de l'hétérogénéité des températures et de la dépendance en la température à la puissance $4\left(T^{4}\right)$ de la fonction de Planck (Dubuisson et Minvielle, 2017). Cependant, là aussi, cela implique des différences qui restent acceptables ${ }^{6}$ si les concepts sont bien interprétés.

\section{Conclusions}

Cet article illustre comment on peut engager un questionnement scientifique avec le public ou des étudiants à partir d'un concept dont chacun a entendu parler, mais qui cache un certain nombre de subtilités à la fois dans sa définition et son estimation. Des expériences menées avec les thermomètres à infrarouge à Eumetsat pendant des journées de type « portes ouvertes » et des séances de travaux dirigés sur la température moyenne de la planète avec des étudiants de $3^{\text {e }}$ année universitaire ont montré que le dialogue pouvait s'amorcer facilement. On a également montré le peu de dépendance des résultats aux choix méthodologiques et donc la robustesse du concept de température moyenne de la planète et du réchauffement observé.

6. Pour se donner un ordre de grandeur de la différence, on peut calculer la moyenne arithmétique de deux températures $274 \mathrm{~K}\left(1^{\circ} \mathrm{C}\right)$ et $302 \mathrm{~K}\left(29^{\circ} \mathrm{C}\right)$ qui est $288 \mathrm{~K}\left(15^{\circ} \mathrm{C}\right)$ et la moyenne arithmétique des températures à la puissance 4 qui correspond à une température de $\left(273^{4}+302^{4}\right)^{1 / 4} \approx 289 \mathrm{~K}\left(16^{\circ} \mathrm{C}\right)$

\section{Bibliographie}

BIPM, 2012. International vocabulary of metrology - Basic and general concepts and associated terms / Vocabulaire international de métrologie - Concepts fondamentaux et généraux et termes associés, $3^{\mathrm{e}}$ édition, Version 2008 avec corrections mineures, Joint Committee for Guides in Metrology. Disponible à l'adresse https://www.bipm.org/utils/common/documents/jcgm/JCGM_200_2012.pdf

Cowtan K., Way R.G., 2014. Coverage bias in the HadCRUT4 temperature series and its impact on recent temperature trends. Q. J. R. Meteorol. Soc., 140, 1935-1944. doi: 10.1002/qj.2297

Dubuisson P., Minvielle F., 2017. Température et rayonnement infrarouge. La Météorologie, 99, 17-24. doi: 10.4267/2042/63586

Hartmann D.L., Klein Tank A.M.G., Rusticucci M., Alexander L.V., Brönnimann S., Charabi Y., Dentener F.J., Dlugokencky E.J., Easterling D.R., Kaplan A., Soden B.J., Thorne P.W., Wild M., Zhai P.M., 2013. Observations: Atmosphere and Surface. In: Climate Change 2013: The Physical Science Basis. Contribution of Working Group I to the Fifth Assessment Report of the Intergovernmental Panel on Climate Change [T.F. Stocker, D. Qin, G.-K. Plattner, M. Tignor, S.K. Allen, J. Boschung, A. Nauels, Y. Xia, V. Bex, P.M. Midgley (eds.)]. Cambridge University Press, Cambridge, UK and New York, NY, USA.

Kent E.C., Kennedy J.J., Smith T.M., Hirahara S., Huang B., Kaplan A., Parker D.E., Atkinson C.P., Berry D.I., Carella G., Fukuda Y., Ishii M., Jones P.D., Lindgren F., Merchant C.J., Morak-Bozzo S., Rayner N.A., Venema V., Yasui S., Zhang H., 2017. A call for new approaches to quantifying biases in observations of Sea Surface Temperature. Bull. Am. Meteorol. Soc., 98, 1601-1616. doi: 10.1175/BAMS-D-15-00251.1

Moisselin J. M., Schneider M., Canellas C., Mestre 0., 2002. Les changements climatiques en France au XXe siècle : Études des longues séries homogénéisées de données de température et de précipitations. La Météorologie, 54, 33-42. doi:10.4267/2042/36233

Rohde R., Muller R.A., Jacobsen R., Muller E., Perlmutter S., Rosenfeld A., Wurtele J., Groom D., Wickham C., 2013a. A new estimate of the average Earth surface land temperature spanning 1753 to 2011. Geoinfor. Geostat.: An Overview, 1:1. doi:10.4172/2327-4581.1000101

Rohde R., Muller R.A., Jacobsen R., Perlmutter S., Rosenfeld A., Wurtele J., Curry J., Wickham C, Mosheet S., 2013b. Berkeley Earth temperature averaging process. Geoinfor. Geostat.: An Overview, 1:2. doi:10.4172/gigs.1000103 